\title{
Erratum to: Growth Behavior of Cubic Boron Nitride (cBN) Phase in B-C-N Film Deposited on Si Substrate with Non-Uniform Ion Flux
}

\author{
Seung-Min Lee, Tae-Yeon Seong, Wook-Seong Lee, Young-Joon Baik, and Jong-Keuk Park
}

Erratum to: Met. Mater. Int., Vol. 19, No. 3, pp. 591-595 (2013)

doi: 10.1007/s12540-013-3031-9

On page 591, first paragraph, 3rd to 10th line, the following sentence should be now read as: In contrast to the diamond thin film with sp3-bonding which has been deposited by chemical vapor deposition (CVD) with a mixed gas of carbon source and hydrogen at a high temperature over $800{ }^{\circ} \mathrm{C}$, the $\mathrm{cBN}$ phase has been mainly synthesized by physical vapor deposition (PVD) with an energetic ion source at a lower temperature below $600^{\circ} \mathrm{C}$, as in the case of transition metal (carbo) nitride coatings [14]. 